\title{
A new index to monitor temporal and long-term variations of the equatorial electrojet by MAGDAS/CPMN real-time data: $E E$-Index
}

\author{
T. Uozumi ${ }^{1}$, K. Yumoto ${ }^{1}$, K. Kitamura ${ }^{2}$, S. Abe ${ }^{1}$, Y. Kakinami ${ }^{1}$, M. Shinohara ${ }^{1}$, A. Yoshikawa ${ }^{1}$, H. Kawano ${ }^{1}$, \\ T. Ueno ${ }^{3}$, T. Tokunaga ${ }^{3}$, D. McNamara ${ }^{4}$, J. K. Ishituka ${ }^{5}$, S. L. G. Dutra ${ }^{6}$, B. Damtie \\ V. Doumbia*8, O. Obrou ${ }^{8}$, A. B. Rabiu ${ }^{9}$, I. A. Adimula ${ }^{10}$, M. Othman ${ }^{11}$, \\ M. Fairos ${ }^{11}$, R. E. S. Otadoy ${ }^{12}$, and MAGDAS Group ${ }^{1}$ \\ ${ }^{1}$ Space Environment Research Center, Kyushu University, 6-10-1 Hakozaki, Fukuoka, Japan \\ ${ }^{2}$ Tokuyama College of Technology, Gakuendai, Shunan, Yamaguchi 745-8585, Japan \\ ${ }^{3}$ Department of Earth and Planetary Sciences, Kyushu University, 6-10-1 Hakozaki, Fukuoka, Japan \\ ${ }^{4}$ Manila Observatory, Bldg. at Ateneo de Manila University Campus, Quezon City, Philippines \\ ${ }^{5}$ Observatorio de Ancón, Instituto Geofísico del Perú, Carretera Panamericana Norte Km. 43.5, Ancón, Peru \\ ${ }^{6}$ National Institute for Space Research (INPE), 12227-010 Sao Jose dos Campos, SP, Brazil \\ ${ }^{7}$ Department of Physics, Bahir Dar University, Bahir Dar, PB 79, Ethiopia \\ ${ }^{8}$ Laboratorire de Physique de l'Atmosphere, University of Cocody, BPV34 Abidijan, Cote d'Ivoire \\ ${ }^{9}$ Department of Physics, Federal University of Technology, PMB 704, Akure, Ondo State, Nigeria \\ ${ }^{10}$ Department of Physics, University of Ilorin, Ilorin, Kwara State, Nigeria \\ ${ }^{11}$ National Space Agency, Ministry of Science, Technology and Innovation, 62100 Putrajaya, Malaysia \\ ${ }^{12}$ Department of Physics, University of San Carlos, Cebu City, Philippines
}

(Received August 27, 2007; Revised February 20, 2008; Accepted March 28, 2008; Online published August 4, 2008)

\begin{abstract}
A new index, $E E$-index ( $E D$ st, $E U$, and $E L$ ), is proposed to monitor temporal and long-term variations of the equatorial electrojet by using the MAGDAS/CPMN real-time data. The mean value of the $H$ component magnetic variations observed at the nightside (LT $=18-06$ ) MAGDAS/CPMN stations along the magnetic equatorial region is found to show variations similar to those of Dst; we defined this quantity as EDst. The EDst can be used as a proxy of $D s t$ for the real-time and long-term geospace monitoring. By subtracting EDst from the $H$ component data of each equatorial station, it is possible to extract the Equatorial Electrojet and Counter Electrojet components, which are defined as $E U$ and $E L$, respectively.
\end{abstract}

Key words: $E E$-index, equatorial electrojet, counter electrojet, MAGDAS/CPMN, real-time monitoring, $D s t$ index, space weather.

\section{Introduction}

The "equatorial electrojet" (EEJ) is observed near the dayside magnetic dip equator as an enhanced magnetic variation superposed on the low-latitude $S q$ (e.g., Forbes, 1981). The EEJ field is caused by the ionospheric current flowing along the narrow channel $\left(\sim \pm 3^{\circ}\right.$ in latitudinal range) of the enhanced ionospheric conductivity (Cowling conductivity), which is formed along the dayside dip equator. Many investigations have been made on this phenomenon from the early stage of geomagnetism (e.g., Chapman, 1951; Onwumechilli, 1997), because it is the most typical and dominant phenomenon showing on ordinary magnetograms obtained at stations in the vicinity of the magnetic dip equator. Despite the wealth of information obtained from these investigations, many open questions regarding EEJ still remain such as (1) the occurrence mechanism of day-to-day and long-term variations of EEJ amplitude, (2) penetration mechanisms of DP2-ULF range disturbances from the solar wind region to the magnetic equator and its relation to the EEJ disturbances, and (3) mechanisms

Copyright (c) The Society of Geomagnetism and Earth, Planetary and Space Sciences (SGEPSS); The Seismological Society of Japan; The Volcanological Society of Japan; The Geodetic Society of Japan; The Japanese Society for Planetary Sciences; TERRAPUB. of ionosphere-thermosphere coupling and its influence on EEJ variations (e.g., Rastogi, 1992; Kobea et al., 1998; Yumoto and the MAGDAS Group, 2006). In recent years, the importance of the study of equatorial phenomena is increasing from the aspect of the space weather.

Quasi-periodic magnetic perturbations of EEJ with a period of about half- to a few-hours are commonly recognized as DP 2 (Nishida, 1968). DP 2 is basically understood as a magnetic perturbation that is closely correlated with the variation of the interplanetary magnetic field (IMF), especially in its north-south component. One of the unique features of the equatorial phenomena is the enhancement of magnetic perturbations caused by the Cowling conductivity. This effect appears on various period ranges, including not only that of DP 2 (Kikuchi et al., 1996) but also that of ULF (Shinohara et al., 1997). The monitoring of EEJ is important for the study of the solar windmagnetosphere-ionosphere-thermosphere coupling system (e.g., Yumoto and the MAGDAS Group, 2006).

The equatorial "counter-electrojet" (CEJ) is also a wellknown phenomenon, which is observed as a depression in the northward magnetic component while a regular EEJ shows an increase (Gouin, 1962). The occurrence mechanism of the CEJ has not yet been clarified sufficiently, 
though its morphological aspects have been accumulated by many researchers (e.g., Mayaud, 1977 and references therein). Rastogi (1974) reported that the occurrence of CEJ is at a maximum when the lunar time is 2.3 and $14.3 \mathrm{~h}(2.2$ and $14.2 \mathrm{~h}$ ) and the local time is around $7 \mathrm{~h}$ (between 15 and $17 \mathrm{~h}$ ). These lunar times correspond to those of maximum negative effect due to the lunar tidal wave, and these CEJ maxima are suggested to be due to the superposition of the lunar semi-diurnal wave over the solar daily wave of $H$. Recently, Kikuchi et al. (2003) proposed that CEJs most likely occur during the substorm recovery phase and that they are caused by the rapid change of the ionospheric electric field generated and transmitted from the IMF, through the mechanism called the over-shielding effect.

The magnetic equatorial region is considered as the terminator of the energy flow associated with the sun-earth coupling system (e.g., Kobea et al., 1998). Thus, it is vital to monitor equatorial geomagnetic phenomena not only to clarify their fundamental occurrence mechanisms but also for space weather studies. In particular, investigation of temporal and long-term variations of equatorial magnetic disturbances associated with equatorial electrojet and counter electrojet requires the construction of a real-time monitoring system. To our knowledge, there is no monitoring system as extensive as the MAGDAS/CPMN, which has a sufficient number of magnetic stations located along the dip equator. In this paper, we propose a new algorithm to achieve the above goals and introduce a new index, the $E E$-Index ( $E D s t, E U$ and $E L$ ), to quantify the scale of magnetic disturbances in the equatorial region using MAGDAS/CPMN real-time data.

\section{Methodology}

The MAGnetic Data Acquisition System of the Circumpan Pacific Magnetometer Network (MAGDAS/CPMN) evolved from the present CPMN for real-time monitoring of the electromagnetic and plasma environment in geospace (Yumoto and the MAGDAS Group, 2006). MAGDAS/CPMN consists of two unique chains of magnetic sta- tions; one is installed densely along the $210^{\circ}$ magnetic meridian, and the other is installed at whole local time sectors along the magnetic equator. In this study, MAGDAS/CPMN 1-min resolution data from four equatorial stations were analyzed to construct a basic algorithm for realtime monitoring of long-term variations of the EEJ and the CEJ.

Figure 1(a) shows a superposed plot of the relative magnetic variation of the $H$ component data $E R_{S}(m)$ (definition given just below) obtained for 1 month (Dec. 1-31, 2006), at the MAGDAS equatorial stations: Addis Ababa $\left(\mathrm{AAB}\right.$ : Dip Lat. $=0.57^{\circ} ;$ GMLON $\left.=110.47^{\circ}\right)$, Davao (DAV: $-0.65^{\circ} ; 196.54^{\circ}$ ), Ancon (ANC: $0.74^{\circ} ; 354.33^{\circ}$ ) and Eusebio (EUS: $-7.03^{\circ} ; 34.21^{\circ}$ ). The " $S$ " and " $m$ " in " $E R_{S}(m)$ " refer to a station and a point of time in UT, respectively. In order to obtain $E R_{S}(m)$, the median value of the $H$ component data, which is determined for the period from the start time of the observation to the end time of $E R_{S}(m)$, was subtracted from the original magnetic data for each station. It was found (not shown) that the median value, which was calculated from all the data in the abovestated interval, could estimate the non-disturbed nighttime ambient level. The determination by shorter-period data might cause some jumps in the base level among different periods (it might occur in particular during disturbed times when large magnetic storms occurred frequently). Thus the median value was calculated for the period from the start time of the observation to the end of the $E R_{S}(m)$. In the future the subtracting offset value will be corrected by taking into account the secular variation of the base level, though the value is currently determined as a constant.

Figure 1(b) plots the superposed $H$ component perturbation field data that was observed during the night time (LT $=18-06)$ at each station: $\left.E R_{S}(m)\right|_{\mathrm{LT}=18-06}$. The solid thick curve (orange) in Fig. 1(b) is the Dst value for the same plot interval. This Dst was provided in real-time on the website of the World Data Center for Geomagnetism, Kyoto (http://swdc234.kugi.kyoto-u.ac.jp/dst_realtime/). In this figure, Dst was graphically shifted downward so as to

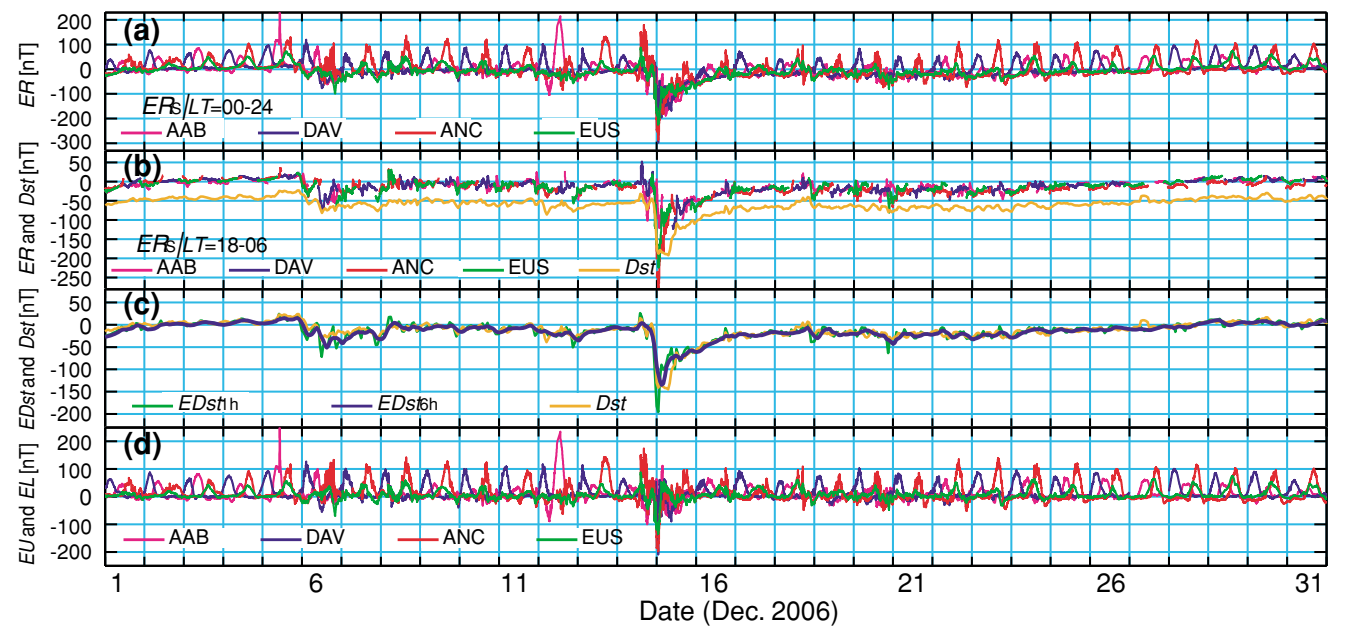

Fig. 1. One month plot of (a) relative magnetic variation of the $H$ component $\left(\left.E R_{S}\right|_{\mathrm{LT}=00-24}\right)$ observed by MAGDAS/CPMN equatorial network stations, (b) relative night time (LT=18-06) variation of the $H$ component $\left(\left.E R_{S}\right|_{\mathrm{LT}=18-06}\right)$ and the real-time $D s t$, (c) $E D s t_{1 \mathrm{~h}}, E D s t_{6 \mathrm{~h}}$ and the real-time $D s t$, and (d) $E U$ and $E L$ for MAGDAS/CPMN equatorial network stations, during one month of December 2006. 
avoid overlap with MAGDAS/CPMN data. It is noticed that the nighttime variation of each station was almost aligned with each other and formed a common base magnetic variation. On the other hand, by comparing Fig. 1(a) and 1(b), it can be confirmed that the daytime EEJ component of each station bulged out independently from the base magnetic variation. With this presentation, it is evident that the base magnetic variation $\left.E R_{S}(m)\right|_{\mathrm{LT}=18-06}$ varies closely with the traditional Dst (Sugiura, 1963). This similarity will be further examined later in this section.

Here we define "EDst (Equatorial Disturbance in storm time)" as follows; at first, the relative nighttime (LT $=18-$ 06) variation of the $H$ component data is determined for each station: $\left.E R_{S}(m)\right|_{\mathrm{LT}=18-06}$ is calculated by the method that was defined above. For the second step, the mean value of the magnetic variations among stations, when they are located in the nighttime sector $(\mathrm{LT}=18-06)$, are calculated at each minute:

$$
\operatorname{EDst}_{1 \mathrm{~m}}(m)=\frac{\left.\sum_{\left.S\right|_{\mathrm{LT}}=18-06} E R_{S}(m)\right|_{\mathrm{LT}=18-06}}{\left.N(m)\right|_{\mathrm{LT}=18-06}}
$$

where the sum operation is carried out for the stations which are located in the nighttime sector (LT $=18-06$ ): $\left.S\right|_{\mathrm{LT}=18-06} .\left.N(m)\right|_{\mathrm{LT}=18-06}$ represents the number of stations which are located in the nighttime sector (LT $=18-$ $06)$ at a specified time point of $m$. In case none of the stations was located in the nighttime sector, $E D s t_{1 \mathrm{~m}}(\mathrm{~m})$ takes a blank value (for the period of the plot, at least one station was located in nighttime sector throughout the interval). 18-06 LT is adopted as the nighttime in the present algorithm. It is noted that the seasonal variation of the nighttime duration takes a minimum value at the equatorial region compared with other latitudes. This means that diurnal variation of the EEJ was almost confined within the 0618 LT independently of any seasons, that the effect of the daytime ionospheric current is not significant for the period of 18-06 LT, and that the nighttime magnetic variation can be utilized as the base level for the EEJ and CEJ. This situation has been confirmed by the DAV data observed from the period of July 2005 to March 2006 (not shown here).

"EDst $t_{1 \mathrm{~h}}$ " is one-hour average of EDst ${ }_{1 \mathrm{~m}}$. In Fig. 1(c), $E D s t_{1 \mathrm{~h}}$ is plotted the green curve. The blue and orange curves in Fig. 1(c) are 6-h running averages of EDst $t_{1 \mathrm{~h}}$ (labeled $\left.E D s t_{6 \mathrm{~h}}\right)$ and the real-time $D s t$, respectively. $D s t$ is shifted $+42 \mathrm{nT}$ to adjust the base level. It is confirmed that the EDst and Dst exhibited the similar temporal variation. It is noticed that $E D s t_{6 \mathrm{~h}}$ is less deviated from $D s t$ than $E D s t_{1 \mathrm{~h}}$. Correlation coefficients between $E D s t_{6 \mathrm{~h}}$ and $D s t$, and that between $E D s t_{1 \mathrm{~h}}$ and $D s t$, which were calculated for the 1-month period of December 2006, were 0.93 and 0.88 , respectively. We have also found that the correlation coefficient between the low-pass-filtered $E D s t_{6 \mathrm{~h}}$ and the low-pass-filtered $D s t$ with the cut-off-period of 2 days was 0.98 is larger than the above-mentioned 0.93 .

In order to examine how the number of used nighttime stations affects the quality of $E D s t_{1 \mathrm{~h}}$, here we try all available combinations of the nighttime stations to calculate $E D s t_{1 \mathrm{~h}}$, calculate the correlation between the thus obtained
$E D s t_{1 \mathrm{~h}}$ and $D s t$, and calculate the standard deviation of $\left(E D s t_{1 \mathrm{~h}}-D s t\right)$. When $E D s t_{1 \mathrm{~h}}$ is calculated from one nighttime station, the correlation coefficient and the standard deviation are in the range of $0.83-0.92$ and $7.12-$ $12.2 \mathrm{nT}$, and their mean values are 0.88 and $10.6 \mathrm{nT}$, respectively. For the case of two nighttime stations, the coefficient and the standard deviation are in the range of $0.91-$ 0.99 and 3.16-11.1 nT, and their mean values are 0.95 and $6.71 \mathrm{nT}$, respectively. For the case of three nighttime stations (in the present analysis, the $\mathrm{AAB}, \mathrm{ANC}$, and EUS group was the available combination), the coefficient and the standard deviation are 0.98 and $6.60 \mathrm{nT}$, respectively. This examination shows that the correlation coefficient between $E D s t_{1 \mathrm{~h}}$ and $D s t$ tends to increase and the standard deviation of (EDst $\left.t_{1 \mathrm{~h}}-D s t\right)$ tends to decrease, as the number of nighttime stations increases.

Figure 1(d) shows the relative magnetic perturbation of each station, which was calculated by subtracting $E D s t_{6 \mathrm{~h}}$ from the $H$ component data. In this calculation, $E D s t_{6 \mathrm{~h}}$ was converted to 1-min resolution data by interpolating the original $E D s t_{6 \mathrm{~h}}$ through application of the FIR (finiteduration impulse response) low-pass interpolating method. It is considered that the positive and negative deviations represent the EEJ and CEJ components, respectively. We define those two component as $E U_{S}(m)$ and $E L_{S}(m)$, respectively. For example, when the $E U$ and $E L$ components are derived for the DAV station, those values are expressed as $E U_{\mathrm{DAV}}(m)$ and $E L_{\mathrm{DAV}}(m)$, respectively. Inspection of these data reveals that the EEJ intensity varies from day to day; moreover, the peaks and dips of each station are not in-phase with other stations. For example, the intensity of EEJ at the DAV station (blue line) and at the ANC station (red line) attained local maxima on Dec. 6 and 8, respectively. The mechanism of the 2-day separation is one of the open questions concerning EEJ. In some cases, it will be necessary to correct the secular variation of a base level to determine $E D s t_{6 \mathrm{~h}}$ for properly extracting the $E U$ and $E L$ component.

In order to examine whether the above-stated procedure properly extracts the EEJ/CEJ component from the original magnetic perturbation and to examine if the obtained $E U / E L$ indices can be utilized for the monitoring of the long-term variation of EEJ/CEJ, we have analyzed the spectral characteristics of the long-term variation of $E U / E L$. The data observed at the DAV station during the period from July 1, 2005 to March 4, 2006 were analyzed. In this period, because MAGDAS/CPMN equatorial network had not been established and data from other stations were not available, $D s t$, instead of EDst, was subtracted from the DAV data. Figure 2(a) and 2(b) shows the Dst and DAV $H$ component data for the above interval, respectively. From this plot it can be seen that the EEJ component is occasionally drowned out by geomagnetic storms, and by looking at the raw data it is hard to see the day-to-day variation of the EEJ during certain periods. However, as shown in Fig. 2(c), this visibility problem can be corrected if $D s t$ is subtracted from the $H$ component data. This is plotted as (DAV $H)-(D s t)$. Despite the disturbance of a geomagnetic storm, the dayto-day variations and oscillations of EEJ intensity can be easily seen in Fig. 2(c). 
(a)

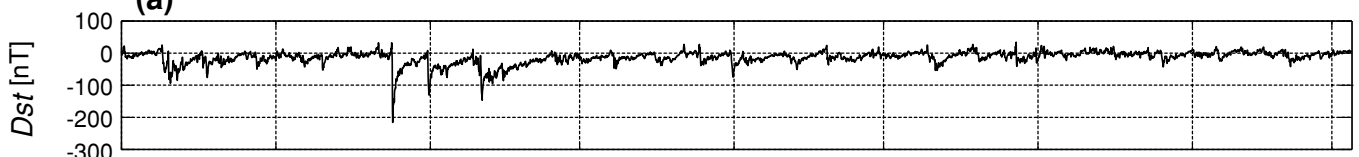

(b)

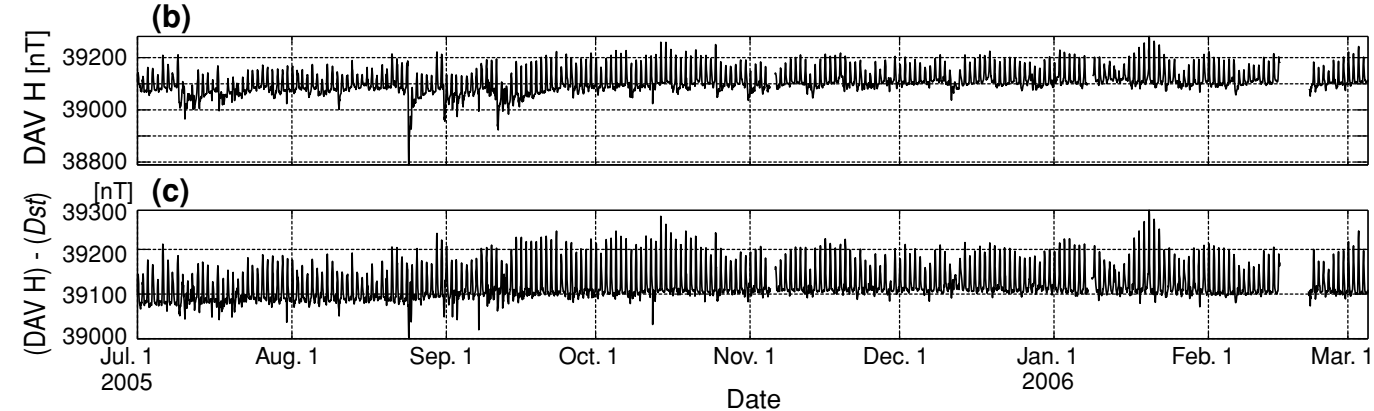

Fig. 2. Long-term variation of (a) Dst, (b) $H$ component observed at DAV (DAV $H$ ), and (c) (DAV $H$ )- $(D s t$ ), during the period from July 1 , 2005 to March 4, 2006.

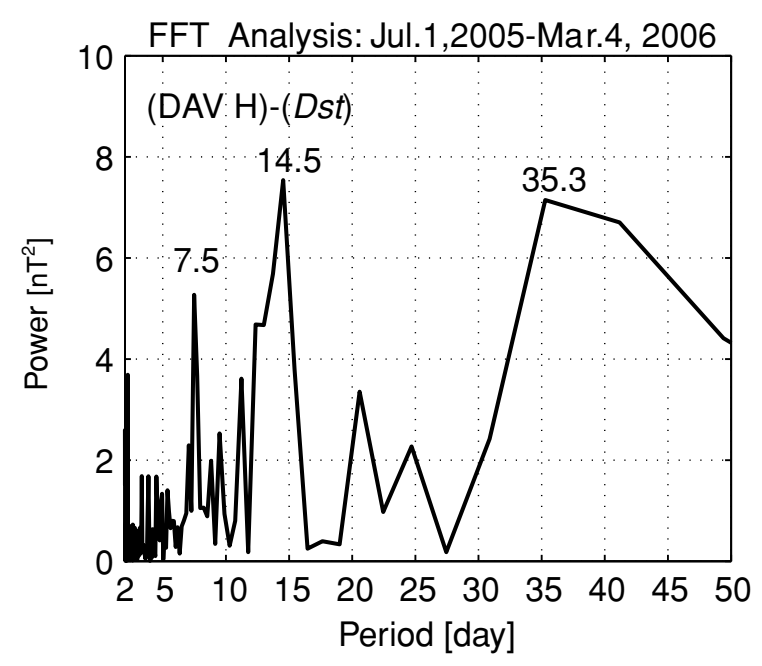

Fig. 3. Power spectrum of the subtracted $H$ component data at DAV [(DAV $H)-(D s t)]$ during the period from July 1, 2005 to March 4, 2006.

Figure 3 shows the power spectrum of the subtracted $H$ component data at DAV; (DAV H)- $(D s t)$ during the period from July 1, 2005 to March 4, 2006. It is recognized that there are at least three dominant peaks at $7.5,14.5$, and 35.3 day (these dominant peaks were visually identified in the figure). Table 1 shows the list of dominant period peaks of various parameters. In the tables, (DAV $H$ ) refers to the original (without $D s t$ subtraction) $H$ component data at DAV. IMF $\left(B_{X}, B_{Y}, B_{Z}\right.$ and $\left.B_{T}\right), V_{\mathrm{SW}}, T_{\mathrm{SW}}$, and $N_{\mathrm{SW}}$ are the interplanetary magnetic field, solar wind velocity, temperature, and number density, which were observed by the ACE spacecraft. $P_{\mathrm{D}}$ and $\epsilon$ are the dynamic pressure and the epsilon-parameter, respectively (Perreault and Akasofu, 1978) calculated by using the ACE solar wind data. $F_{10.7}$ represents the flux of the solar radio emission at $10.7 \mathrm{~cm}$ wavelength. The data of $F_{10.7}$ was provided at the website of the Herzberg Institute of Astrophysics (http://www.draoofr.hia-iha.nrc-cnrc.gc.ca/icarus/www/archive.html). It is noteworthy that the above-stated dominant period peaks of 7.5 and 14.5 days, which appeared in the spectrum of (DAV
Table 1. Dominant peak periods detected in the power spectrum for various parameters during the period from July 1, 2005 to March 4, 2006.

\begin{tabular}{|c|c|c|c|c|c|c|c|c|}
\hline \multicolumn{9}{|c|}{ Jul. 1, 2005 - Mar. 4, } \\
\hline Variable & \multicolumn{8}{|c|}{ Peak Period [day] } \\
\hline (DAV H) - (Dst) & 7.5 & & & 14.5 & & & & 35.3 \\
\hline$(\mathrm{DAV} H)$ & 7.7 & 9.2 & 11.2 & 14.5 & 17.6 & 22.5 & 27.4 & \\
\hline Dst & 7.7 & 9.2 & 11.2 & & 17.6 & 22.5 & 27.4 & \\
\hline $\mathrm{Kp}$ & & 9.2 & & & & & 27.4 & \\
\hline IMF Bx,y,z,T & & 9.2 & 13 & & & & 27.4 & \\
\hline Vsw & & 9.2 & 13. & & & & 27.4 & \\
\hline Tsw & & 9.2 & 13. & & & & 27.4 & \\
\hline Nsw & & 9.2 & 11.2 & & & & 27.4 & 35.3 \\
\hline PD & & 9.2 & 11.2 & & & & 27.4 & 35.3 \\
\hline$\varepsilon$ & & 9.2 & & & & 22. & & \\
\hline $\mathrm{F} 10.7$ & & & & & & 22. & & 35.3 \\
\hline
\end{tabular}

$H)-(D s t)$, did not have their roots in the geomagnetic activity indices of $D s t$ and $K p$ nor any parameters such as IMF, $V_{\mathrm{SW}}, T_{\mathrm{SW}}, N_{\mathrm{SW}}, P_{\mathrm{D}}, \epsilon$, and $F_{10.7}$, although the peak at 35.3 days was also identified in $N_{\mathrm{SW}}, P_{\mathrm{D}}$, and $F_{10.7}$. On the other hand, the original $H$ component data contained the same spectrum component as geomagnetic activity indices and solar wind parameters, except 35.3 day.

\section{Discussion}

We have constructed a basic algorithm to monitor in realtime the long-term variations of the (EEJ) and the (CEJ) by analyzing the MAGDAS/CPMN data. We also proposed new $E E$-indices, which consist of $E D s t, E U$, and $E L$, for the monitoring of the equatorial geomagnetic phenomena. The real-time monitoring system of the MAGDAS/CPMN equatorial network stations, which are spaced longitudinally along the dip-equator, will provide a fundamental reference of various geomagnetic phenomena observed in the magnetic equatorial region.

As shown in Fig. 1(c), the mean nighttime variation at each moment (named as E Dst), which were observed at the MAGDAS/CPMN equatorial network stations, was highly correlated with the traditional Dst (Sugiura, 1963). That is, in spite of the smallness of the total number of the nighttime stations (1-3 stations depending on UT) for the ex- 
amination period, EDst was derived in sufficient quality, as shown in the previous section. The correlation between Dst and EDst tends to increase, and the standard deviation of $\left(E D s t_{1 \mathrm{~h}}-D s t\right)$ tends to decrease with increasing number of nighttime stations. When the number of nighttime stations is more than one, the coefficient is larger than $\sim 0.95$, and the standard deviation is smaller than $\sim 7 \mathrm{nT}$. This value corresponds to $\sim 5 \%$ of the normal EEJ amplitude (e.g., Doumouya et al., 2003). This result suggests that $E D s t$ has sufficient quality if more than one nighttime station available for $E D s t$ calculation. When the $E E$-index published, the number of nighttime stations used to calculate $E D s t$ will also be published as index for the validity of EDst. It is noted that more stations on the magnetic equator promise a better accuracy; the MAGDAS/CPMN equatorial network will extend the number of stations in the future.

Using the obtained EDst, the EEJ and CEJ component were derived - and named as $E U_{S}$ and $E L_{S}$ for each equatorial stations $(S)$ - by using only the MAGDAS/CPMN data without referring to provisional/real-time Dst. EDst has consistency with $D s t$, and concurrently derived $E U_{S}$ and $E L_{S}$ are possibly utilized as real-time monitoring of the temporal and long-term variation of the EEJ and CEJ phenomena. These are useful not only for clarifying their fundamental mechanism but also for studying the space weather. The statistical consistency between $D s t$ and ED $D t$ should be verified successively as long-term data will be accumulated. The realtime $E E$-index will be published on the website at "http://www.serc.kyushu-u.ac.jp/", and the rules for public usage of the $E E$-index will also be informed there.

Although EDst is similar to Dst (as stated above), they are not identical, and it is important to construct the monitoring system, which is independent of provisional/realtime Dst, as stated below. The behavior of the magnetic variations which are observed at the equatorial stations are different from those of the low-latitude Dst stations (for example, the effect of $S q$ are different). Some portion of the dissimilarity between EDst and Dst could be caused by the different behavior of the magnetic variations (and others could be caused by the day-night asymmetry; EDst is derived only from nighttime data). The $S q$ component, which was deduced from the quiet-day magnetic variation for each Dst station, was subtracted in deriving Dst; however, the subtraction might not be perfect, especially in the provisional/real-time data. That is, it is possible that there exists some remnant of the $S q$ component on Dst, and the remnant could affect the $E E$-index if we use $D s t$ in deriving the $E E$-index. In addition to the above, the independent system has advantage to react flexibly to the change in the observational situation of each station (offset level, secular variation of the base level, etc.).

In order to derive the EEJ and CEJ component, the nighttime level in the neighboring days has been commonly referred to as the base level. For example, Fambitakoye and Mayaud (1976) used linearly interpolated values connecting neighboring days' midnight values as the basis in deriving the EEJ and CEJ components. Rigoti et al. (1999) defined the reference (base) level as follows: "At midnight, the traces are close to a straight line, showing no signifi- cant magnetic variation. Hence, the midnight value or the mean between the preceding and subsequent midnight values can be taken as the zero reference level for the diurnal variation". The presented method is an extension of such traditional algorithms and is basically consistent with them. The improvement of our algorithm is that it takes into account the temporal variation of the nighttime level affected by external magnetic variations. The traditional base level in the literature did not include the magnetic variation that occurred between both ends of the interpolated period. It is noted that our new algorithm is not just a mathematical extension of the traditional procedure but that it has a geophysical meaning in that it subtracts Dst. The spectrum analysis (Table 1) revealed that subtraction of $D s t$ (or $E D s t$ ) from the $H$ component data removed external magnetic disturbances. This procedure has an advantage of enabling the extraction of the EEJ and CEJ components even under disturbed geomagnetic conditions and to realizing continuous monitoring of the EEJ and CEJ.

As shown in Table 1, subtracted $H$ component data at an equatorial station: (DAV $H)-(D s t)$, showed 7.5-, 14.5-, and 35.3-day periodicities and did not contain any spectrum components for periods longer than the 2 days included in the geomagnetic indices and solar wind parameters, except for the 35.3 days (it is a topic of future research to examine if the periods 7.5 and 7.7 days have a common source or not). It is reasonable that the same result could be obtained if we adopted EDst instead of Dst on deriving the spectrum pattern of (DAV $H)-(D s t)$, because, as examined in the previous section, magnetic variation with periods longer than 2 days was highly correlated between EDst and Dst. This suggests that by subtracting $E D s t$ from the $H$ component data at each equatorial station, local effects can be deduced, which are interpreted in this paper as the long-term variation of EEJ. The specifics of the local effects have not been fully understood. This is a topic for future research.

One possible cause of those local effects is the interaction between the upper atmosphere and the ionosphere through the planetary or the Rossby normal mode wave (Forbes et al., 1995). The power spectrum shown in Fig. 3 exhibited similar pattern compared with the results presented by Forbes and Leveroni (1992). They found spectrum peaks at 16 and 34 days. In the present analysis, the spectrum peak of the 35.3-day period appearing in the variation of (DAV $H)-(D s t)$ corresponded to those of $N_{\mathrm{SW}}, P_{\mathrm{D}}$; however, this result might not directly point to $N_{\mathrm{SW}}$ or $P_{\mathrm{D}}$ as a cause of the ground 35.3-day variations. As suggested by Forbes and Leveroni (1992), this could be the effect of the solar radiation $\left(F_{10.7}\right)$ on the ionosphere and, consequently, on EEJ. The spectral analysis for $F_{10.7}$ radiation represented the existence of 22.5- and 35.3-day peaks. It supports the above suggestion. We note that the $E E$-index may include the effect of long-term oscillations in the latitudinal location of EEJ (e.g., Osborne, 1962). The frequencies of such oscillations would show themselves in the FFT results, but it is a topic of future research to identify the cause of each FFT component related to EEJ found in this paper. The realtime monitoring of EEJ by MAGDAS/CPMN will enable and promote the investigation of mechanisms that result in the long-term variations within the coupled system of the 
atmosphere-ionosphere.

In this paper, we have proposed a new geomagnetic index, the $E E$-index, which is derived from the data of the MAGDAS/CPMN equatorial network. This network allows for the first time real-time monitoring of geomagnetic phenomena in the magnetic-equatorial region. This index will provide information that should clarify the situation of solar-geospace coupling and atmosphere-ionosphere coupling along the magnetic equatorial region. Long-term and continuous study of the $E E$-index should greatly expand the understanding of the fundamental mechanisms pertaining to various equatorial geomagnetic phenomena.

Acknowledgments. We thank World Data Center for Geomagnetism Kyoto for providing geomagnetic indices: Dst and $K p$. We thank the ACE MAG and SWEPAM instrument team and the ACE Science Center for providing the ACE data. We thank the the Herzberg Institute of Astrophysics for providing $F_{10.7}$ solar radiation data. The PI of MAGDAS/CPMN project, K. Yumoto, SERC, Kyushu Univ. very much appreciates 30 organizations and Co-investigators around the world for their ceaseless cooperation and contribution to the MAGDAS/CPMN project. Financial supports were provided by Japan Society for the Promotion of Science (JSPS) as Grant-in-Aid for Overseas Scientific Survey $(15253005,18253005)$ and for publication of scientific research results $(188068,198055)$.

\section{References}

Chapman, S., The equatorial electrojet as detected from the abnormal electric current distributions above Huancayo, Peru, and elsewhere, Arch. Meteorol. Geophys. Bioclimatol., A4, 368-390, 1951.

Doumouya, V., Y. Cohen, B. R. Arora, and K. Yumoto, Local time and longitude dependence of the equatorial electrojet magnetic effects, $J$. Atmos. Solar-Terr. Phys., 65, 1265-1282, 2003.

Fambitakoye, O. and P. N. Mayaud, Equatorial electrojet and regular daily variation $S_{\mathrm{R}}-\mathrm{I}$. A determination of the equatorial electrojet parameters, J. Atmos. Terr. Phys., 38, 1-17, 1976.

Forbes, J. M., The Equatorial Electrojet, Rev. Geophys. Space Phys., 19, 469-504, 1981.

Forbes, J. M. and S. Leveroni, Quasi 16-day oscillation in the ionosphere, Geophys. Res. Lett., 19, 981-984, 1992.

Forbes, J. M., M. E. Hagan, S. Miyahara, F. Vial, A. H. Manson, C. E. Meek, and Y. I. Portnyagin, Quasi 16-day oscillation in the mesosphere and lower thermosphere, J. Geophys. Res., 100, 9149-9163, 1995.

Gouin, P., Reversal of the magnetic daily variation at Addis Ababa, Nature, 193, 1145-1146, 1962.
Kikuchi, T., H. Lühr, T. Kitamura, O. Saka, and K. Schlegel, Direct penetration of the polar electric field to the equator during a DP 2 event as detected by the auroral and equatorial magnetometer chains and the EISCAT radar, J. Geophys. Res., 101, 17,161-17,173, 1996.

Kikuchi, T., K. Hashimoto, T.-I. Kitamura, H. Tachihara, and B. Fejer, Equatorial counterelectrojets during substorms, J. Geophys. Res., 108(A11), 1406, doi:10.1029/2003JA009915, 2003.

Kobea, A. T., C. Amory-Mazudier, J. M. Do, H. Lühr, E. Houngninou, J. Vassal, E. Blanc, and J. J. Curto, Equatorial electrojet as part of the global circuit: a case-study from the IEEY, Ann. Geophys., 16, 698-710, 1998.

Mayaud, P. N., The equatorial counter-electrojet-a review of its geomagnetic aspects, J. Atmos. Terr. Phys., 39, 1055-1070, 1977.

Nishida, A., Geomagnetic DP 2 fluctuations and associated magnetospheric phenomena, J. Geophys. Res., 73, 1795-1803, 1968.

Onwumechilli, C. A., The Equatorial Electrojet, Gordon and Breach Science Publishers, Netherlands, 1997.

Osborne, D. G., Position and movement of the equatorial electrojet over Ghana, J. Atmos. Terr. Phys., 24, 491-502, 1962.

Perreault, P. and S.-I. Akasofu, A study of geomagnetic storms, Geophys. J. R. Astron. Soc., 54, 547, 1978.

Rastogi, R. G., Lunar effects in the counter electrojet near the magnetic equator, J. Atmos. Terr. Phys., 36, 167-170, 1974.

Rastogi, R. G., Critical problems of equatorial electrojet, Adv. Space. Res., 12, 13-21, 1992.

Rigoti, A., F. H. Chamalaun, N. B. Trivedi, and A. L. Padilha, Characteristics of the Equatorial Electrojet determined from an array ofmagnetometers in N-NE Brazil, Earth Planets Space, 51, 115-128, 1999.

Shinohara, M., K. Yumoto, A. Yoshikawa, O. Saka, S. I. Solovyev, E. F. Vershinin, N. B. Trivedi, J. M. Da Costa, and The $210^{\circ}$ MM Magnetic Observation Group, Wave characteristics of daytime and nighttime Pi 2 pulsations at the equatorial and low latitudes, Geophys. Res. Lett., 24, 2279-2282, 1997.

Sugiura, M., Hourly values of equatorial Dst for the IGY, Goddard Space Flight Center, Greenbelt, Maryland, NASA Rept., X-611-63-131, June, 1963.

Yumoto, K. and the MAGDAS Group, MAGDAS project and its application for space weather, in Solar Influence on the Heliosphere and Earth's Environment: Recent Progress and Prospects, edited by N. Gopalswamy and A. Bhattacharya, ISBN-81-87099-40-2, 399-405, 2006.

T. Uozumi (e-mail: uozumi@serc.kyushu-u.ac.jp), K. Yumoto, K. Kitamura, S. Abe, Y. Kakinami, M. Shinohara, A. Yoshikawa, H. Kawano, T. Ueno, T. Tokunaga, D. McNamara, J. K. Ishituka, S. L. G. Dutra, B. Damtie, V. Doumbia, O. Obrou, A. B. Rabiu, I. A. Adimula, M. Othman, M. Fairos, R. E. S. Otadoy, and MAGDAS Group 